\title{
Diacronie
}

Studi di Storia Contemporanea

$N^{\circ} 13,1 \mid 2013$

Contrabbandieri, pirati e frontiere: per una storia delle pratiche informali nell'America Centrale (XVII-XXI

secolo)

\section{Le Pirate, acteur interstitiel de la colonisation européenne dans les Caraïbes (XVIe-XVIIIe siècles)}

\section{Caroline Laurent}

\section{(2) OpenEdition}

\section{Journals}

Édition électronique

URL : http://journals.openedition.org/diacronie/655

DOI : 10.4000/diacronie.655

ISSN : 2038-0925

Éditeur

Association culturelle Diacronie

Référence électronique

Caroline Laurent, « Le Pirate, acteur interstitiel de la colonisation européenne dans les Caraïbes (XVIeXVIIle siècles) », Diacronie [En ligne], № 13, 1 | 2013, document 1, mis en ligne le 01 avril 2013, consulté le 20 avril 2019. URL : http://journals.openedition.org/diacronie/655 ; DOI : 10.4000/ diacronie. 655 


\title{
Diacronie
}

\section{Le Pirate, acteur interstitiel de la colonisation européenne dans les Caraïbes (XVIe-XVIIIe siècles)}

\author{
Caroline LAURENT *
}

Cet article analyse l'évolution de la perception de la piraterie caribéenne au moment de la colonisation européenne, et tente d'expliquer la profusion des représentations qui lui sont associées de nos jours dans l'imaginaire collectif. La prise en considération des contextes politique, économique et social européens permet ainsi de mettre en lumière les enjeux liés à la piraterie dans le bassin caribéen à l'ère dite des "Grandes Découvertes". D'autre part, une analyse de la situation sociale et politique du pirate engage à repenser l'histoire de la piraterie "par le bas", à la manière de Marcus Rediker, Peter Linebaugh et Christopher Hill. La notion d'“interstice" est alors convoquée dans une tentative de meilleure compréhension de la figure du pirate.

\section{Introduction}

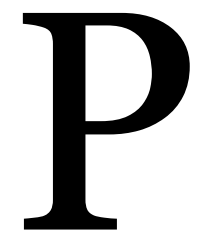

irate, corsaire, flibustier ? Trois termes pour désigner une même catégorie sociale, versant maritime du bandit de grands chemins, qui se distinguent les uns des autres par les divers degrés de légalité qui leur sont conférés par les États européens selon les contextes diplomatiques. Les flibustiers se métamorphosent ainsi en véritables bras armés des puissances européennes (particulièrement britannique, espagnole et française) dans cette zone caribéenne qui est considérée comme terra nullius, “territoire sans maître”, jusqu'au XVIIIe siècle, temps de la mainmise effective des empires sur le bassin caribéen. Car il est alors nécessaire pour les empires d'instituer un ordre dans ces espaces insulaires, et 
particulièrement les bras de mer qui les fragmentent.

Avant que le bassin caribéen ne devienne le haut lieu de la flibuste occidentale au XVIe et surtout au XVIIe siècle, la Manche et la mer du Nord figurent les principales scènes des exactions des " gueux de mer », principalement hollandais. De même, de l'autre côté du globe et à la même époque, le phénomène de la piraterie a la vie belle en mer de Chine; on le retrouve également près d'un millénaire plus tôt dans la Méditerranée Antique, avant d'y faire à nouveau fortune entre le XIe et le XIIIe siècle.

L'objectif de cet article est de comprendre comment le pirate caribéen est passé du statut d'avant garde instrumentalisée de la colonisation européenne informelle dans les Amériques, à celui du "forban", banni par les empires, pour finalement échouer dans l'imaginaire. Il s'agit d'une approche globale, tant dans l'espace que dans le temps, pour tendre vers la mise en évidence de certaines caractéristiques générales du pirate. Nous reviendrons brièvement dans un premier temps sur les conditions qui poussent nombre d'Européens à grossir les rangs de la flibuste et à sillonner les eaux caribéennes à partir du XVIe siècle. L'évolution nette du regard porté par les pouvoirs impériaux du « vieux continent » sur la piraterie caribéenne entre le XVIe et le XVIIIe siècle révèle ainsi l'envergure de ce phénomène. Ce processus sera étudié de manière indirecte, notamment à travers la notion d'interstice, qui semble particulièrement féconde au regard de la figure du pirate, pour qui la marge constitue un véritable refuge. Enfin, nous tenterons de mettre au jour certains aspects ayant conduit la figure du pirate caribéen a s’inscrire de façon si prégnante dans l’imaginaire collectif occidental.

\section{Une identité floue, composée de multiples facettes}

Avant toute chose, il est nécessaire de distinguer corsaires, flibustier et pirates. Un certain nombre de traits distinctifs caractérisent ces quatre termes. Cependant, ces distinctions ne reposent pas tant sur l'activité à laquelle se livrent ces marins, qu'aux cadres légaux que projettent sur eux les pouvoirs qui leur sont contemporains.

Le corsaire est celui à qui une autorité royale a délivré ce que l'on appelle une « lettre de course », ou encore « lettre de marque », le plus souvent en temps de guerre, ou de guerre économique qui ne dit pas son nom. Celle-ci stipule les conditions dans lesquelles le corsaire peut agir, à savoir : les nations qu'il peut attaquer, et la période durant laquelle ces attaques peuvent être menées. Le corsaire peut donc être assimilé à un mercenaire à la solde d'une nation.

Les caractéristiques du flibustier sont légèrement moins nettes. Celui que l'on désigne par un mot dérivé du hollandais est donc un marin qui, muni de lettres de 
course ou non, se livre à des expéditions à des fins lucratives, et dont l'activité est généralement tolérée par les pouvoirs. Effectivement, avant que le bassin caribéen ne devienne le haut-lieu de la flibuste occidentale au XVIe et surtout au XVIIe siècle, la Manche et la mer du Nord figurent les principales scènes des exactions des « gueux de mer », principalement hollandais. Ils lègueront leur nom à leurs successeurs caribéens : le mot vrijbuiter ("libre-butineur") mute en freebooter dans la langue anglaise, avant de finalement passer au français avec le terme "flibuste". Le phénomène de la flibuste caribéenne connaît son âge d'or au XVIIe siècle, avec en particulier la constitution de véritables enclaves flibustières dans l'archipel.

Celui que l'on qualifie de pirate ne possède pas de lettre de course et agit en conséquence dans un cadre strictement illégal, c'est pourquoi il est aussi désigné par le terme «forban ", et on le rencontre surtout à partir du XVIIIe siècle (bien que ces tentatives de découpage chronologique semblent assez vaines). Mais son activité en tant que telle ne diffère guère de celle du corsaire et, dans une moindre mesure, de celle du flibustier.

Pour conclure cette tentative d'éclaircissement, peut-être peut-on rapidement évoquer les boucaniers ${ }^{1}$, qui sont liés aux flibustiers. Effectivement, les boucaniers peuvent être vus comme le versant terrestre de la flibuste du fait que «les mêmes hommes [cumulent] très souvent les deux activités, car piller ports et navires se [pratique] après avoir chassé le bœuf sauvage ou le sanglier d'Hispaniola ${ }^{2}$. Ils répondent ainsi, par leurs activités, aux besoins des navires en vivres. D’autre part, « [ils] se [rapprochent] des flibustiers par leur origine ; beaucoup [sont] des marins ayant déserté les équipages des flottes espagnoles (...) $»^{3}$, d'où la confusion qui peut régner entre ces derniers.

On remarque là que ce qui différencie corsaire, flibustier et pirate ne réside pas tant dans l'activité-même de ces marins, que dans la perception des pouvoirs à leurs égards, et le statut de légalité qu'ils leur confèrent ou non. Nous laissons pour le moment en suspend cette question de l'évolution des tentatives d'encadrement et même de répression de l'activité piratique, qui sera abordée plus largement par la suite.

La question de l'origine de la piraterie caribéenne peut contribuer à éclairer les causes de l'importance que ce phénomène revêt dans l'aire caribéenne à l'époque

\footnotetext{
${ }^{1}$ Terme qui provient du mot "boucan", sorte de foyer sur lequel les Amérindiens font fumer la viande, technique que les colons européens reprennent à leur compte lorsqu'ils s'installent dans les îles.

${ }^{2}$ BUTEL, Paul, Les Caraïbes au temps des flibustiers, Paris, Éditions Aubier Montaigne, 1982, p. 79.

${ }^{3}$ BUTEL, Paul, op. cit., p. 79.
} 
coloniale. Ainsi, malgré la tentation de la rapprocher d'autres phénomènes éloignés dans le temps et l'espace mais a priori analogues, la piraterie caribéenne fait preuve d'une particularité vis-à-vis de ses consœurs asiatique et méditerranéennes, ou encore de la mer du Nord : elle exerce son activité dans un espace "autre", qui ne correspond pas avec celui dont elle est issue. La piraterie caribéenne est déterritorialisée et en ce sens, profondément hétéroclite.

Si cette piraterie est "caribéenne" puisque le Golfe du Mexique, les Antilles et les côtes d'Amérique centrale constituent, aux XVIe et XVIIe siècles, son "terrain de chasse" de prédilection, elle est pourtant bel et bien majoritairement européenne. Protestants et autres hérétiques fuyant l'intolérance religieuse, repris de justice déportés aux Indes, marins de profession et soldats fuyant les mauvais traitements subis sur les navires des flottes impériales, engagés ${ }^{4}$ faisant face aux désillusions de leur nouvelle vie dans le "nouveau monde" et se soustrayant aux dures conditions de travail : se sont tous des exilés qui se joindront aux équipages pirates en quête d'une vie meilleure. Cette dernière affirmation appelle cependant à être nuancée, dans la mesure où il n'est pas rare d'y croiser également des "marrons" (ces esclaves noirs qui s'échappent des plantations), dont certains parviennent à se hisser jusqu'au sommet de la hiérarchie flibustière, mais également parce que les flibustiers peuvent parfois compter sur le soutien d'Amérindiens issus des différentes tribus caraïbes, au sujet desquels nous nous devons d'émettre quelques réserves en les considérant davantage comme des alliés potentiels que comme de véritables pirates.

La majorité des marins grossissant les rangs flibustiers de la Caraïbe sont donc majoritairement issus des bas-fonds des sociétés européennes. Une proportion nonnégligeable d'entre eux fuient une vie régie par la misère, l'asservissement, et par l'absence de perspectives puisque déterminées dès la naissance (la mobilité sociale est alors pour ainsi dire inexistante). Par ailleurs, les puissances européennes n 'hésitent pas à envoyer massivement aux Amériques les criminels, vagabonds et autres marginaux qui n'ont pas leur place dans les métropoles et qu'on destine ainsi à la colonisation et à l'exploitation des terres récemment conquises outremer; le gouvernement de Cromwell envoie notamment à la Jamaïque les vagabonds et les prisonniers de Newgate, la célèbre prison londonienne. Enfin, le XVIe siècle est aussi le siècle des guerres de religion en Europe, opposant Catholiques et Réformés protestants, qu'on appelle aussi "Huguenots" en France. Ces conflits religieux dépassent le cadre

\footnotetext{
${ }^{4}$ Colons s'engageant à travailler jusqu'à une dizaine d'années sur une plantation ou une exploitation quelconque afin de rembourser leur voyage depuis l'Europe, avancé par le propriétaire de ladite plantation ou exploitation.
} 
strict de l'Europe et sont perceptibles dans les colonies, à travers la course à la colonisation, marquant telle ou telle terre du sceau des religions catholique ou protestante. Car les Amériques représentent un véritable enjeu tant pour l'Eglise catholique, soucieuse d'étendre son aire d'influence outre-Atlantique, que pour les Réformés, voyant dans ces nouveaux territoires des espaces vierges à conquérir car représentant des refuges potentiels pour les Protestants alors persécutés en Europe. Par exemple, plusieurs groupes de groupes radicaux protestants anglais, tels les Diggers, les Quakers, ou encore les Ranters, s'exilent dans les colonies américaines en quête de terres plus favorables où ils pourraient exercer leur foi et vivre selon leurs principes sans avoir à subir de persécution. On retrouverait ainsi la trace de ces radicaux dans les îles caribéennes aux XVIe et XVIIe siècles : «dans les années 1630, la Providence Island Company arrach[e] une île aux Espagnols pour en faire un refuge offert aux dissidents persécutés, ainsi qu'une base pour tenter de briser le monopole des catholiques de la région $»^{5}$.

Enfin, c'est avec la "découverte" des Amériques que s'ouvre «la phase mercantiliste du capitalisme» à un niveau international, et il se trouve que «le mercantilisme [est] l'une des conditions majeures qui [favorise] l'apparition de la piraterie en déplaçant les travailleurs maritimes de leurs ports d'attache, maintenant les petits négociants hors du commerce maritime, et rendant la survie économique dans les colonies encore plus difficiles lorsque l'importation de denrées alimentaires ou d'autres biens de première nécessité [est] strictement contrôlée ${ }^{6}$. Ce processus, encadré par les États, et en particulier grâce aux Compagnies de commerce européennes qui établissent des monopoles nationaux sur les produits importés depuis les Amériques, doit donc bien être lié au phénomène de la piraterie ; « les pirates des Caraïbes s'opposent clairement au contrôle du travail et des biens souvent violent exercé par les compagnies mercantiles nationales, et à ce qu'ils considèrent comme étant des inégalités "tyranniques"7.

Il apparaît clairement que, dans tous les cas de figure, ce passage vers l'illégalité et

\footnotetext{
${ }^{5}$ WEBER, Dominique, «Hobbes, les pirate et les corsaires. Le "Léviathan échoué" selon Carl Schmitt », in Astérion, 2/2004, URL: <http://asterion.revues.org/document94.html >, [consulté le 10 fevrier 13].

${ }^{6}$ «[...] mercantilism was one of the major conditions that helped create piracy by dislocating maritime workers from their familiar ports, keeping smaller traders out of the shipping business, and making economic survival in colonies even more difficult when the import of foodstuffs or other deemed necessities were strictly controlled». DAWDY, Shannon Lee, BONNI, Joe, «Towards a General Theory of Piracy», in Anthropological Quaterly, 85, 3/2012, p. 681.

7 «[...] the pirates of the Caribbean explicitly opposed the often violent control of labor and goods by nationalist mercantile companies and what they viewed as "tyrannical" inequalities ", ibidem, p. 677.
} 
la marginalité, résulte d'un choix libre et conscient. De plus, outre l'appât du gain, on remarque que c'est la fuite d'un système particulier qui pousse ces individus à franchir la ligne rouge. Que ce soient les sociétés européennes, où l'ascension sociale est pour ainsi dire inexistante et où la foi catholique est imposée, les sociétés de plantations en Amérique, ou encore les systèmes carcéral, militaire, ou économique, ces hommes fuient des systèmes qui encadrent leurs vies et qui les maintiennent dans des situations misérables, ou pour le moins considérées comme non-acceptables, pour grossir les rangs de la piraterie.

On comprend donc aisément que "les Indes", avec le lot de mythes et de légendes qui en émanent déjà, ne cessent d'attirer des hommes, jeunes pour la plupart, et mus par le désir de briser le cours de leur vie, qui peut leur sembler à bien des égards inexorable.

\section{Une évolution du regard porté sur la piraterie à l'époque moderne}

Il semble opportun de replacer la flibuste caribéenne dans son contexte historique et économique global, après avoir esquissé le contexte social. En effet, comprendre les rapports qui liaient la piraterie et le pouvoir exerçant son autorité dans les zones où cette dernière évolue nécessite que l'on s'attarde un moment sur les mutations mêmes que connaît alors ce pouvoir. En l'occurrence, ce que nous désignons par "pouvoir" correspond en particulier aux monarchies espagnoles, anglaises et française, qui se lancèrent progressivement dans une course effrénée ayant pour objectif la maitrise des terres récemment découvertes et l'exploitation des richesses que celles-ci peuvent offrir. La période à laquelle on s'intéresse ici, et qui s'étend de la "découverte" des Amériques par les Européens (1492) à la fin du XVIIIe siècle, coïncide exactement avec la période que les historiens ont appelée "époque moderne". Un des facteurs ayant conduit les historiens à segmenter l'histoire européenne de telle façon que l'on considère dorénavant la période qui débute au XVIe siècle comme étant bien différente des siècles la précédent, réside dans les mutations politiques qui eurent lieu à cette époque, qui débouchent sur la formation des états-nations européens. Ce sont ces mutations politiques et juridiques qui expliquent en partie la raison pour laquelle les pirates sont sujets à de telles différences de traitement entre les débuts de la colonisation européenne des Amériques et les indépendances.

Nous avons vu que la flibuste est pendant un temps tolérée (au XVIIe siècle surtout). Cela coïncide effectivement avec les intérêts de certaines puissances européennes (notamment française et britannique), du fait des grandes rivalités 
existant entre ces dernières et l'Espagne. De fait, l'auto-proclamation de celle-ci en tant qu'unique puissance possédant le droit de commercer depuis les Amériques, d'ailleurs légitimée par la bulle papale Inter Caetera de 1494, contrarie ainsi les ambitions d'expansion commerciale et coloniale de ses voisins européens, qui voient d'un mauvais œil les richesses du continent américain se déverser dans la seule péninsule ibérique. De même, selon les nations, les distinctions entre corsaire, flibustier et pirate n'existent pas nécessairement, et le "souci de légalisme » de la part de certaines d'entres elles quant à ces marins enclins à "l'activité piratique" n’a tout simplement pas lieu d'être :

Un souci de légalisme à pondérer, bien sûr, d'un fort coefficient d'hypocrisie, de la part de toutes les parties concernées, variant au gré des intérêts et des rapports de force - car un conflit majeur sous-tendait toutes les tentatives de règlement, et par avance les ruinait : celui qui opposait les tenants de la liberté des mers, comme la France, et ceux qui, arc-boutés aux bulles pontificales, tenaient pour nulle et nonavenue toute lettre de marque dans les zones de leur monopole, et pour acte de piraterie la simple présence dans ces eaux d'un bateau étranger ${ }^{8}$.

Côté espagnol, il apparaît donc que dès les débuts de la colonisation, la moindre incursion d'un navire battant pavillon étranger dans les eaux caribéennes suffise à le situer en dehors de tout cadre légal, et donc à le désigner comme "pirate ». Côtés français et britannique surtout, et dans une moindre mesure côtés hollandais et portugais, la démarcation est moins nette.

On peut considérer que l'Espagne aura les coudées relativement franches en Amérique tout au long du XVIe siècle, même si elle subit occasionnellement les attaques de corsaires des nations ennemies qui, déjà, sillonnent les eaux caribéennes. Au même moment, la France est confrontée aux guerres de religion qui ne s'achèveront qu'en 1598 avec la promulgation de l'Édit de Nantes par Henri IV. On comprend, dés lors, que la constitution d'un empire s'étendant outremer est loin d'être la priorité de la France, qui doit alors régler ses conflits internes, et délègue cette tâche à des armateurs privés souhaitant y faire fortune. Les prétentions de l'Espagne à monopoliser le territoire américain ne peuvent rester lettre morte très longtemps. Très vite, une tension s'installe entre François Ier, roi de France de 1515 à 1547, et Charles Quint, empereur du Saint Empire Germanique et roi d'Espagne sous le nom de Carlos I de 1519 à 1555. Les navires français qui naviguent dans les eaux caribéennes sont ainsi légitimés par François Ier, conscient des avantages à tirer de ces incursions sur les voies

\footnotetext{
${ }^{8}$ LE BRIS, Michel, D'or, de rêves et de sang. L'épopée de la flibuste, 1494-1588, Paris, Hachette, 2001, p. 62.
} 
commerciales espagnoles : «face aux prétentions de l'Espagne, la diplomatie française [développe] une agressivité entretenue par les intérêts liés à la course [qui enrichit le marché français], à la pêche de la morue et à la découverte d'un nouveau passage vers les îles à épices »". C'est donc ainsi que la course française prend pied dans le bassin caribéen.

Ils sont des centaines, alors, des milliers, partis de tous les ports du Ponant, qui trafiquent ainsi, pêchent, ou font la course, le long des côtes du Nouveau Monde, sans le moindre égard pour les bulles papales, si nombreux et si hardis qu'Espagnols et Portugais feront bientôt preuve à leur endroit d'une rare férocité générant en retour des représailles non moins terribles, qui feront pour longtemps des mers des Caraïbes les plus violentes du globe ${ }^{10}$.

L'Angleterre et les Pays-Bas revendiquent à leur tour le droit de circuler librement sur les mers. Les années du règne d'Elisabeth Ière d'Angleterre (1558-1602) se trouvent être les années durant lesquelles l'Angleterre se hisse littéralement au rang de grande puissance maritime, en grande partie grâce à l'opiniâtreté des privateers, ces armateurs privés, sur lesquels s'appuiera Elisabeth pour conquérir à son tour les océans. A tel point que l'Angleterre du XVIIe siècle se voit bientôt désigner comme une "nation de pirates" dont les "chiens de mers", entrainés, entre autres, par John Hawkins et plus tard Francis Drake, deviendront la phobie des Espagnols. Les activités des flibustiers sont alors plus que légitimées par le pouvoir royal anglais, puisque celui-ci encourage, voire même organise discrètement ces expéditions destinées à ébranler la toute puissance espagnole, qui est occupée à tenter d'apaiser ses propres conflits internes : en 1566, elle se heurte à l'opposition des Pays-Bas qui réclament leur indépendance, elle subit un grave revers en 1588 avec la déroute de l'Armada Invincible face à la flotte anglais, elle perd le Portugal en 1640 suite à la révolte de Lisbonne, et est alors empêtrée dans une situation économique catastrophique.

Si l'Angleterre, la France et la Hollande ne sont pas encore officiellement présentes en tant que puissances dans la Caraïbe au XVIe siècle et dans la première moitié du XVIIe siècle, la présence de leurs sujets représente une avant-garde non négligeable dans cette zone, qui permet à plus long terme l'implantation de colonies soutenues et voulues par ces États. Cette avant-garde est d'autant plus encouragée qu'elle nuit à l'Espagne catholique, ce qui n'est pas sans déplaire à ses voisins européens qui prennent conscience des multiples profits que peuvent offrir les Amériques. Un trafic

\footnotetext{
${ }^{9}$ BUTEL, Paul, op. cit., p. 33 .

${ }^{10}$ LE BRIS, Michel, op. cit., p. 22. 
interlope très intense se développe dans l'espace caraïbe. Au XVIIe siècle, les navires hollandais et anglais prolifèrent dans ces eaux, qui leur sont pourtant théoriquement interdites par les Espagnols, tenant à leur monopole : " la compagnie hollandaise [profite] de la faiblesse des réactions espagnoles pour développer [...] une grande activité, dont l'histoire constitue un des premiers chapitres de la geste de la flibuste aux Antilles au XVIIe siècle $»^{11}$.

L'économie caraïbe connaît au cours des XVIe, XVIIe et XVIIIe siècles plusieurs cycles correspondant aux cultures alors les plus rentables. Dans un premier temps, c'est la culture du tabac qui séduit de nombreux petits propriétaires désireux de prospérer rapidement. Toutefois, la concurrence anglaise au cours du XVIIe siècle affaiblit la demande. Ces petits propriétaires ne pourront se mettre à la culture de l'indigo, « et encore moins à celle de la canne à sucre, car ces cultures [exigent] beaucoup de capitaux et beaucoup de terre $»^{12}$. La culture du sucre se généralise peu à peu, engageant ces sociétés vers le système de plantations, sur lesquelles on fait de plus en plus appel à la force de travail des esclaves importés d'Afrique. Avec le passage aux sociétés de plantations et donc le passage à un nouveau type d'économie, on assiste à un phénomène double: d'une part, les engagés sont délaissés et vont grossir les groupes de flibustiers qui sillonnent l'espace caribéen et, de l'autre, les pouvoirs métropolitains engagent une répression de plus en plus contraignante et violente afin d'assurer la sécurité de leurs territoires.

En effet, le tournant vers l'espace maritime entrepris par l'Angleterre s'accompagne d'un renforcement du pouvoir de l'État, qui entend faire respecter ses lois en tous lieux où s'exerce sa souveraineté. En 1651, le premier Act of Navigation est promulgué. Le Léviathan de Hobbes est publié la même année, et est à cet égard très représentatif d'une nouvelle conception du pouvoir, qui deviendra absolu au XVIIIe siècle, qui cherchera à étendre "l'empire de la souveraineté étatique jusqu'à l'élément maritime lui-même $»^{13}$.

L'Angleterre suit cette voie en affirmant chaque fois davantage sa souveraineté sur les mers et en manifestant sa volonté d'étendre son empire dans l'aire américaine, et notamment caribéenne et sur les côtes des actuels Etats-Unis, où elle envoie plusieurs colonies. La sécurité de ces zones devient alors un enjeu majeur : l'Angleterre ne peut plus tolérer que les flibustiers attaquent impunément des navires, et qu'ils nuisent à l’implantation programmée de leurs colonies. C'est la raison pour laquelle une loi visant

\footnotetext{
${ }^{11}$ BUTEL, Paul, op. cit., p. 43.

${ }^{12}$ Ibidem, p. 150.

${ }^{13}$ WEBER, Dominique, op. cit., p. 8.
} 
à la « suppression effective de la piraterie » sera finalement mise en place en 1698 : the Act for the More Effectual Suppression of Piracy ${ }^{14}$. Les flibustiers sont, dés lors, explicitement considérés comme des pirates, et c'est à partir de ce basculement qu'ils seront véritablement réprimés et considérés comme hostes humani generis : " c'est l'idée d"“eaux territoriales" qui commence précisément à être élaborée au XVIIIe siècle ${ }^{15}$.

Les pirates qui ne se rallient pas à la cause nationale en intégrant les flottes officielles n'ont donc plus droit de cité dans les eaux caribéennes et deviennent des hors-la-loi. La France n'est pas en reste car Louis XIV réaffirme dans le même temps son autorité sur ses possessions dans les Antilles en créant la "Compagnie des Indes occidentales". L'administration directe royale remplace donc le régime des propriétaires privés.

Les traités d'Utrecht (1713) qui mettent fin à la guerre de Succession d'Espagne entérinent de façon définitive la répression de la flibuste, et donc de la piraterie, qui n'est plus utile aux États qui sont désormais suffisamment bien établis dans la Caraïbe et qui constitue une véritable menace au regard des alliances diplomatiques contractées entre les différentes couronnes européennes. Les pirates sont donc passés du statut d'alliés indispensables, à celui de "forbans". La course reste cependant tolérée, alors que parallèlement les flibustiers qui se refusent à rentrer dans le rang sont persécutés.

Les ennemis du pirate l'ont lentement déconnecté de l'ordre social, le présentant comme ennemi de tous les individus, des propriétaires de biens, des colonies, de l'empire, du roi, de la nation britannique, du monde des nations, et finalement de toute l'humanité ${ }^{16}$.

Parallèlement à cette modification de la perception des pouvoirs européens, un changement opère parmi ces "forbans": si au temps de la "Renaissance » de la piraterie (autour de 1450-1650) les pirates et privateers "s'identifiaient eux-mêmes par l'origine nationale et la religion », à l'époque de son « Âge d'or » (autour de 16501730), «ils se proclament "sans-nation" » et se métamorphosent en véritables

14 LINEBAUGH Peter, REDIKER Marcus, L'hydre aux mille-têtes: l'histoire cachée de l'Atlantique révolutionnaire, Paris, Éditions Amsterdam, 2008, p. 223 [ed. or. The ManyHeaded Hydra: Sailors, Slaves, Commoners, and the Hidden History of the Revolucionary Atlantic, Boston, Beacon Press, 2001].

${ }^{15}$ WEBER, Dominique, op. cit., p. 9.

${ }^{16}$ REDIKER, Marcus, Pirates de tous les pays, l'âge d'or de la piraterie Atlantique (1716-1726), Paris, Libertalia, 2008, p. 232 [Villains of All Nations. Atlantic Pirates in the Golden Age, (17161726), Boston, Beacon Press, 2005]. 
apatrides ${ }^{17}$. Un double processus d'exclusion semble se mettre en place : les sociétés européennes bannissent les pirates, qui dans le même temps rejettent toute appartenance nationale. Une nuance nécessaire doit être apportée, évoquée entre les lignes dans les paragraphes précédents : le pirate caribéen des débuts de la colonisation n'est pas le même que celui de l'ère préindustrielle.

C'est donc tant pour des facteurs politiques qu'économiques que les flibustiers se voient progressivement repoussés de l'aire caribéenne, et dans le même temps rejetés et considérés comme des forbans par les États. Ceux-ci engagent de véritables campagne de diabolisation destinées à s'allier le soutien des masses populaires, « déconnectant » ainsi les pirates de leur réalité sociale et politique, les contraignant à naviguer dans les interstices des eaux territoriales européennes qui se déploient au XVIIIe siècle, les obligeant à se réfugier dans les failles du/des système(s), les reléguant finalement aux marges de l'histoire coloniale. Tous les liens ne sont cependant pas rompus. Il se trouve que le pirate conserve sa place dans l'imaginaire collectif, bien que son image ait pu se modifier, se moduler au cours du temps.

\section{Une figure interstitielle qui permet la multiplication des représentations}

On peut discerner parmi les flibustiers et pirates diverses facettes. Bien qu'on les associe sous une étiquette commune, les pirates constituent a priori un ensemble hétérogène, que ce soit par les trajectoires personnelles qui les conduisent à rejoindre les rangs de la flibuste ou encore par leur manière d'exercer cette activité, hormis les différences liées à l'évolution générale de la piraterie aperçues précédemment (à savoir, par exemple, s'ils participent au commerce des esclaves noirs ou non, s'ils entendent faire respecter l'égalité de traitement sur leur navire ou non, s'ils n'attaquent que certains pavillons ou bien tous sans distinction, etc). C'est sans doute une des raisons pour lesquelles certaines analyses divergent tant, voire même s'opposent : si certains préfèrent mettre en évidence la liberté et surtout l'indépendance qui guidait ces marginaux envers et contre tout - comme souligne Rediker -, d'autres au contraire choisissent de considérer cette minorité comme ayant représenté une frange violente de la population, que l'on peut associer à une forme de banditisme répréhensible, qui menaçait les intérêts communs. La piraterie, remarquent justement Dawdy et Bonni, « à cause de son ambiguïté morale, donne elle-même lieu à un tissu de fantasmes peu

\footnotetext{
${ }^{17}$ DAWDY, Shannon Lee, BONNI, Joe, op. cit., p. 679.
} 
commun et à des interprétations politiques variées» ${ }^{18}$.

Les multiples représentations associées à la figure du pirate témoignent de l'attrait que ce dernier exerce sur l'imaginaire collectif. Ce que nous appelons "figure du pirate" correspond plus précisément à cette entité historico-culturelle aujourd'hui plus que fantasmée, véhiculant diverses valeurs et représentations qui parfois s'opposent, et prêtant à des interprétations variées. Elle recouvre donc aussi bien la réalité fantasmée que la réalité historique correspondant aux flibustiers et forbans qui sillonnaient les eaux caribéennes à l'époque moderne, avec le lot d'approximations que cela implique. Si elle est encore présente dans les esprits de nos jours, c'est qu'elle a régulièrement été revisitée et réinvestie. Aujourd'hui encore, sa vivacité est notamment perceptible si l'on porte attention, par exemple, à la profusion de supports ludiques destinés aux enfants où elle est mis en scène, mais également aux multiples occurrences du terme " pirate » dans de nombreux domaines (de l'informatique à l'industrie musicale, en passant par la biologie ou encore la politique, pour ne citer que certains d'entre eux).

Le pirate caribéen est très présent dans le domaine culturel, et ce depuis l'époque moderne. Pour ce qui est de la littérature, on rencontre effectivement le pirate dès le XVIIe siècle avec les chroniques et autres récits de voyage. Car « en-dehors des épopées antiques, c'est dans les récits de voyage que paraissent les premières images de ces “tyrans de la mer" " ${ }^{19}$. Parmi ceux-ci, l'Histoire d'aventuriers qui se sont signalés dans les Indes d'Oexmelin (ou Exquemelin) se distingue particulièrement, compte tenu du succès que celui-ci rencontra à sa parution (1678 pour la version néerlandaise). Le retentissement de cette œuvre est tel qu'elle sera traduite en pas moins de quatre langues - allemand (1679), espagnol, anglais, et français - dans les dix années qui suivirent sa première publication. Les pirates ne sont pas étrangers au succès que rencontra le livre d'Oexmelin, étant donné que, chirurgien de profession, celui-ci se joignit lui-même à la flibuste pendant un temps. Ce livre représente même davantage dans l'histoire de la littérature : " la publication d'Exquemelin en 1686, qui est la première histoire française de la flibuste, marque la rupture avec le romanesque baroque et l'entrée du flibustier dans l'orbite de la narration réaliste» ${ }^{20}$. Le pirate devient en peu de temps (moins d'un siècle) un personnage à part entière, faisant ainsi naître un véritable genre littéraire : le roman d'aventures.

\footnotetext{
${ }^{18}$ « Piracy, because of its moral ambiguity, lends itself to quite different material fantasies, and quite different political interpretations ». DAWDY, Shannon Lee, BONNI, Joe, op. cit, p. 676.

${ }^{19}$ MOUREAU, François, Préface in LINON-CHIPON, Sophie, REQUEMORA, Sylvie, Tyrans de la mer: pirates, corsaires et flibustiers, Paris, Presses de l'Université Paris-Sorbonne, 2002, p. 6.

${ }^{20}$ Ibidem, p. 7 .
} 
Véhicule de la mort et de l'injustice, le pirate est aussi le messager de l'au-delà pour le chrétien, d'une vie autre pour le commun des mortels. D'où la fascination que le pirate exerce sur ses victimes - source de romanesque - ou sur l'imaginaire social. L'image du pirate, homme libre, héros sans moralité sinon sans morale, se forme précisément au confluent de l'Âge classique et des Lumières comme un impossible absolu et pourtant réel ${ }^{21}$.

A partir du moment où le pirate fait son entrée dans le champ du romanesque, celui-ci s'éloigne progressivement de son « origine référentielle ${ }^{22}$. Il est dorénavant perçu à travers le prisme littéraire, ce qui contribue à amoindrir la frontière entre le réel et la fiction dans l'imaginaire collectif. A partir du moment où le pirate est "représenté”, que ce soit via la littérature, ou même la peinture, la musique ou encore, plus tard, le cinéma et les jeux vidéos, ce dernier perd de la consistance, au profit de l'image que l'on en donne. L'imaginaire commence à prendre le pas sur la réalité ; d'une part, du fait de la distance spatiale existant entre le lecteur des XVIIIe et XIXe siècles et le pirate de chair et d'os, et d'autre part, du fait de la distance temporelle existant entre celui-ci et le lecteur des XXe et XXIe siècles.

Nous l'avons vu, le pirate caribéen est contraint d'évoluer dans les failles, qu'elles soient juridique (lorsque son statut n'est pas clairement défini par les États), sociale (en ce qu'on peut le considérer comme un "désaffilié" ${ }^{23}$ des sociétés traditionnelles, ayant préféré s'inscrire dans une trame sociale alternative), économique (en se révoltant contre les pratiques liées à l'émergence du mercantilisme), territoriale (étant donné qu'il se réfugie dans des espaces où la force de coercition étatique des puissances européenne se fait moindre, à l'image de l'espace maritime), ou encore historique (le pirate n'a que rarement sa place dans les manuels d'histoire généraux abordant la colonisation européenne des Amériques, malgré le rôle qu'il a pu y jouer). Nous choisissons donc de définir le pirate caribéen comme une « figure interstitielle », qui n'a pas sa place dans ses sociétés d'origine qui sont allées jusqu'à le bannir de l'espèce humaine, et qu'il a fini par rejeter de lui-même. Ce dernier n'avait d'autre choix que de se cantonner à leurs marges et de s'organiser collectivement en des zones "entre-deux", dont le caractère indéfini leur garantissait une relative autonomie et précaire survie. «L'occupation par le pirate d'espaces à la fois dangereux et distants des centres

\footnotetext{
${ }^{21}$ Ibidem.

${ }^{22}$ Ibidem.

${ }^{23} \mathrm{Au}$ sens donné par Robert Castel de la « désaffiliation sociale » dans le premier chapitre de CASTEL, Robert, Métamorphoses de la question sociale, Paris, Folio, 1995.
} 
d'autorité est un élément clé de son image» ${ }^{24}$.

"L’image du pirate est attachée de près à l'espace qu'il occupe ${ }^{25}$. Or, M. Le Bris oppose la mer, «l'empire du mouvant », à la terre ferme, « espace quadrillé par les savoirs et les coutumes ${ }^{26}$. En le faisant apparaître comme semblable aux monstres peuplant les limites du monde connu dans l'imaginaire collectif des temps modernes, un processus de déshumanisation se met en place. L'une des particularités de l'espace maritime réside dans la perception qu'en ont eu les hommes à l'époque des "Grandes Découvertes", perception qui a, par ailleurs, contribué à former l'imaginaire associé aux individus amenés à le parcourir. S'arrêter un temps sur cet espace permet d'envisager d'autres facteurs ayant mené les pirates à évoluer dans les interstices des sociétés européennes.

Outre les particularités géographiques de l'espace insulaire, l'île elle même en tant qu'espace a très tôt exercé une fascination certaine chez les hommes. C'est un espace qui a été à de nombreuses reprises exploitée par des penseurs et écrivains comme lieu de fixation de réalités idéales ou de réalités alternatives à celles que ces derniers connaissaient. Les communautés pirates installées sur les îles caribéennes aux XVIe et XVIIe siècles ont été à plusieurs reprises appelées « utopies pirates ». L'île serait ainsi, plus généralement, un lieu d'aventures, un lieu foncièrement autre où l'imagination rencontre un terreau très fertile. L'espace insulaire caribéen aurait-il donc favorisé, par ses particularités, l'établissement de communautés indépendantes pirates, basées sur des normes alternatives à celles des sociétés européennes, et qu'on a posteriori décrit comme utopiques ? $^{27}$

Gilles Deleuze propose une interprétation philosophique de l'espace insulaire, et plus particulièrement de "l'île déserte", qui enrichit notre réflexion vis-à-vis de notre analyse des espaces qu'habitent, côtoient ou traversent les pirates caribéens. Ainsi, dans un texte intitulé « Causes et raisons de l'île déserte », Deleuze soutient que «pour qu'une île cesse d'être déserte [...] il ne suffit pas qu'elle soit habitée ${ }^{28}$. « L'essence de l'île déserte est imaginaire et non réelle, mythologique et non géographique ${ }^{29}$, et cette distinction retient notamment notre attention en ce qu'elle permet d'interroger «le milieu géographique comme [...] un réservoir de possibilités pour l'action » à la suite de

${ }^{24}$ REDIKER, Marcus, Pirates de tous les pays, l'âge d'or de la piraterie Atlantique (1716-1726), cit., p. 215 .

${ }^{25}$ Ibidem, p. 214.

${ }^{26}$ LE BRIS, Michel, op. cit., p. 80.

${ }^{27}$ Voir BEY, Hakim, T.A.Z, «The Temporary Autonomous Zone», New York City, Autonomedia, 2003, pp.95-147; LAMBORN WILSON, Peter, Pirate Utopias, Moorish Corsairs \& European Renegadoes, New York City, Autonomedia, 2003.

${ }^{28}$ DELEUZE, Gilles, L’̂̀le déserte et autres textes, Paris, Éditions de Minuit, 2002, p. 12.

${ }^{29}$ Ibidem, p. 14. 
Deleuze.

L'île serait donc bien, à la lumière de la pensée deleuzienne, le lieu du recommencement par excellence, celui qui optimiserait à son maximum le rapport qu'entretiennent milieu et action. L'île est aussi facteur de marginalité, par sa séparation d'avec le continent. Mais elle est surtout le lieu où les capacités de recréation de l'homme sont le plus sollicitées et encouragées :

A partir de l'île déserte ne s'opère pas la création elle-même mais la re-création, non pas le commencement mais le re-commencement. Elle est l'origine, mais l'origine seconde. A partir d'elle tout recommence. L'île est le minimum nécessaire à ce recommencement, le matériel survivant de la première origine $[\ldots]^{30}$.

Cela explique peut-être en partie (conjointement au fait que les Caraïbes constituaient alors un carrefour marchand indéniable) la raison pour laquelle, inconsciemment, des pirates des XVIe et XVIIe siècles ont choisi des îles pour se sédentariser de façon temporaire, parvenant ainsi à territorialiser leur altérité et marginalité radicales illustrées par leur mode de vie. Le choix du territoire en question pourrait ne pas être dû qu'à une coïncidence fortuite ; l'île permettrait, peut-être plus qu'aucun autre espace, la constitution de microsociétés dont le mode de fonctionnement diffère radicalement des sociétés déjà établies qui possèdent une inertie telle que toute nouveauté, toute altérité radicale en est exclue et/ou condamnée. L'île pourrait constituer une des conditions ayant permis à ces hommes d'exercer et d'inventer un nouveau mode de vie qui, a posteriori, a conduit certains, rêvant à ces hypothétiques ancêtres qui auraient inauguré une forme primitive de socialisme - voire même d'anarchisme, selon Rediker -, à les considérer comme de véritables « sociétés utopiques ».

Un vaste monde imaginaire s'est construit autour de la figure du pirate, et ce depuis son entrée dans la littérature au XVIIe siècle. Et même plus, c'est un véritable mythe qui s'est progressivement tissé autour du flibustier, un mythe sans cesse réactualisé par ses représentations culturelles, qui se sont d'ailleurs multipliées et propagées avec l'apparition des nouvelles technologies de l'information et de la communication Internet principalement-, où il est suggéré à travers des références directes, surtout iconographiques, mais également à travers le vocabulaire employé. Ne resterait donc du pirate de l'époque moderne qu'un ersatz de celui-ci, qui fertilise l'imaginaire. Les éléments pouvant porter à controverse (tels les contrats liant pirates et pouvoirs qui étaient monnaie courant à une époque) et/ou qui tendraient à nuancer le portrait que

\footnotetext{
${ }^{30}$ Ibidem, p. 16.
} 
l'on fait du pirate dans ses représentations, sont le plus souvent mis de côté, dans la culture populaire, où il n'a pas survécu au cours du temps en tant que figure historique. Effectivement, il semble s'y être peu à peu désincarné, intégré que très récemment (et de façon sporadique, dans le milieu universitaire) dans les processus de transmissions de savoir de façon officielle et conséquente. C'est donc ce qui nous conduit à situer le pirate dans une nouvelle brèche, un nouvel interstice : culturel cette fois-ci.

Doit cependant être souligné le fait que ces dernières années, l'histoire du pirate caribéen est relue à la lumière des nouvelles pratiques liées à l'événement d'Internet et des nouvelles technologies en général, auxquelles il confère un référent culturel majeur. Il participe ainsi à l'élucidation de nouvelles problématiques relatives à des questions d'ordre social, économique ou encore politique. A ce sujet, les travaux de S. L. Dawdy \& J. Bonni, P. Leeson, A. S. Dent et A. M. Galvin pour ne citer qu'eux, pourraient prolonger l'historiographie relative aux pirates proposée par C. R. Pennell en $1998^{31}$.

\section{Conclusion}

Les flibustiers ont joué un rôle crucial dans le processus de colonisation, et notamment dans les Antilles, en ce qu'ils ont très tôt constitué l'avant-garde de l'implantation de colonies, surtout anglaises et françaises, dans cette partie des Amériques. Toutefois, leur rôle doit vite être restreint à cette fonction d'avant-garde par les métropoles qui, à partir du moment où leur pouvoir commence à être bien assis et défini dans cette zone, décident de l’interdiction pure et simple de l'activité flibustière, si difficile à maîtriser, et qui parallèlement s'émancipe des systèmes politico-sociaux européens. Une partie des flibustiers répond à cette attaque en se radicalisant davantage, et c'est à ce moment que la figure du pirate entre en scène.

C'est bien la conjoncture de divers facteurs, situant le pirate comme une "figure interstitielle", dont nous avons tenté de donner un bref aperçu ici, qui explique le fait que les pirates caribéens aient survécu de façon aussi prégnante dans l'imaginaire collectif, occidental du moins, jusqu’à nos jours.

\footnotetext{
${ }^{31}$ PENNELL, Richard C., "Who needs Pirate Heroes ? », in The Northern Mariner/Le Marin du nord, VIII, 2/1998, pp. 61-79.

Voir: LEESON, Peter, The Invisible Hook: The Hidden Economics of Pirates, Princeton, Princeton University Press, 2011; DENT, Alexander Sebastian, «Understanding the war on Piracy, Or Why We Need More Anthropology of Pirate » in Anthropological Quaterly, 85, 3, pp. 659-672; GALVIN, Anne M., "Caribbean Piracies/Social Mobilities : Some Commonalities Between Colonial Privateers and Entrepreneurial "Profiteers" in the 21st Century ", in Anthropological Quaterly, 85, 3, pp. 755-784.
} 


\section{* L'auteur}

Caroline Laurent est titulaire d'un master recherche mention « Espaces, Sociétés, Cultures dans les Amériques » délivré par l'Institut Pluridisciplinaire pour les Études sur les Amériques de l'Université de Toulouse-II le Mirail. Ses recherches concernent la piraterie caribéenne à l'époque coloniale, mais également la piraterie informatique, plus particulièrement les phénomènes de désobéissance civile électronique aux États-Unis et au Mexique avec le mouvement zapatiste. Actuellement étudiante en Anthropologie à l'UTM.

URL: < http://www.studistorici.com/progett/autori/\#Laurent >

\section{Per citare questo articolo:}

LAURENT, Caroline, «Le Pirate, acteur interstitiel de la colonisation européenne dans les Caraïbes (XVle-XVIIle siècles)», Diacronie. Studi di Storia Contemporanea: Contrabbandieri, pirati e frontiere: per una storia delle pratiche informali nell'America Centrale (XVII-XXI secolo), 29/04/2013,

URL:<http://www.studistorici.com/2013/04/29/laurent_numero_13/ >

Diacronie Studi di Storia Contemporanea $\beta$ www.diacronie.it

Risorsa digitale indipendente a carattere storiografico. Uscita trimestrale. redazione.diacronie@hotmail.it

Comitato di redazione: Marco Abram - Jacopo Bassi - Luca Bufarale - Alessandro Cattunar - Elisa Grandi - Deborah Paci - Fausto Pietrancosta - Matteo Tomasoni - Luca Zuccolo 